\title{
Coherent origin of peculiar polarization in radio pulsars
}

\author{
Jarosław Dyks \\ Nicolaus Copernicus Astronomical Center, Bartycka 18, 00-716 Warsaw, Poland \\ email: jinx@ncac.torun.pl
}

\begin{abstract}
The observed polarization of radio pulsars involves several peculiar effects, such as comparable amount of orthogonal polarization modes (OPMs) which often bear the same handedness of circular polarisation $V$. In the average profiles of $\mathrm{B} 1913+16$ and B1933+16, orthogonal jumps of polarization angle (PA) are observed to occur at the maximum $V$, instead of $V=0$. High levels of $V$ are also observed in core components (eg. in B1237+25), where they are accompanied by strong distortions of PA from the rotating vector model. In weakly polarized emission, PA jumps by $45^{\circ}$ are observed in B1919+21 and B0823+26. It is shown that all these peculiarities can be interpreted in a model which assumes coherent addition of waves in natural propagation modes.
\end{abstract}

Keywords. pulsars: general, radiation mechanisms: nonthermal

\section{The nature of orthogonal polarization modes}

The model assumes that pulsars emit a linearly polarized signal $\vec{E}$ that enters some intervening birefringent region, characterised by the polarization basis $\left(\vec{x}_{1}, \vec{x}_{2}\right)$ (Fig. 1a). The basis is misaligned with $\vec{E}$ by the incident angle $\psi_{\text {in }}$. The incident signal is split into two natural mode waves $\vec{E}_{1}$ (dotted) and $\vec{E}_{2}$ (solid) that are orthogonally polarized with respect to each other. While propagating through the region, the waves acquire a relative phase lag $\Delta \phi$ (Fig. 1a). After leaving the region, the waves combine, i.e. are added coherently. Thus, the model is empirical and similar to the Faraday rotation effect.

The two main parameters, $\psi_{\text {in }}$ and $\Delta \phi$, are drawn from statistical distributions $N_{\psi \text {,in }}$ and $N_{\Delta \phi}$, supposedly produced by the stochastic nature of the emission and propagation processes. The distributions represent the spread of values as recorded in single pulse observations at a fixed pulse longitude. As described in Dyks (2017, hereafter D17), the peak of $N_{\psi \text {,in }}$ is determined by the relative orientation of the magnetic field in the emission and intervening regions. The peak position and width of $N_{\Delta \phi}$ are treated as free parameters. In general, arbitrary pairs of $\psi_{\text {in }}$ and $\Delta \phi$ produce elliptically polarized radiation, with the ellipse major axis, hence the $\mathrm{PA}$, at any orientation with respect to the main polarisation directions $\vec{x}_{1}$ and $\vec{x}_{2}$. However, when the lag distribution $N_{\Delta \phi}$ encompasses the value of $\Delta \phi=\pi / 2$, the situation is different. As shown in Fig. 1, for $\Delta \phi=\pi / 2$, only the ellipses parallel to either $\vec{x}_{1}$ or $\vec{x}_{2}$ are produced, regardless of the $\psi_{\text {in }}$ value. Note that in Fig. 1 the same phase lag of $\pi / 2$ is applied to two different incident angles: $\psi_{\text {in }}=65^{\circ}$ (Fig. 1a) and $25^{\circ}$ (1b). In this way two observed orthogonal modes are produced, as represented by the ellipses $\mathrm{M}_{1}$ and $\mathrm{M}_{2}$. Importantly, these observed modes should be discerned from the natural orthogonal propagation modes that are presented by the waves $m_{1}$ and $m_{2}$. The coherently produced orthogonal modes $\left(M_{1}\right.$ and $\left.M_{2}\right)$ are elliptical, and can easily have the same handedness of $V$, which is the case for the values of $\psi_{\text {in }}$ used in Fig. 1. 

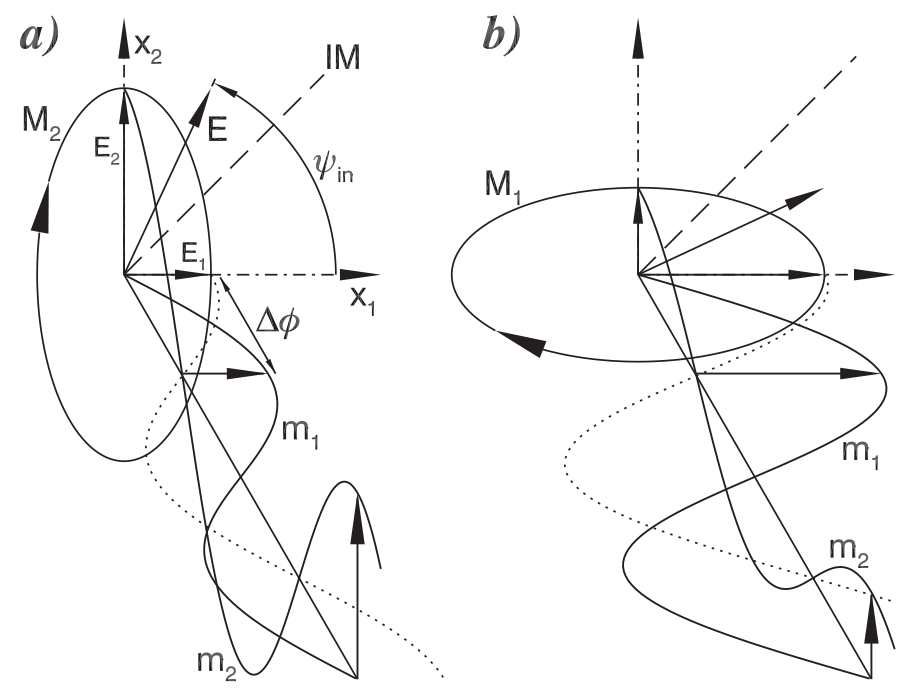

Figure 1. The origin of observed orthogonal polarization modes, represented by the ellipses $M_{1}$ and $M_{2}$, as a coherent sum of the phase lagged natural mode waves $m_{1}$ and $m_{2}$. Note the same handedness of both modes.

For phase lags in the vicinity of $\pi / 2$, the orientation of the modal ellipses is always close to $\vec{x}_{1}$ or $\vec{x}_{2}$, so the corresponding (modal) PAs vastly outnumber other PA values (see Fig. 4 in D17). Here 'other values' means PAs for $\Delta \phi \neq \pi / 2$ and arbitrary $\psi_{\text {in }}$ that correspond to ellipses misaligned with respect to $\vec{x}_{1}$ or $\vec{x}_{2}$. These nonmodal PA values contribute to wide PA distributions that accompany the narrow modal PA peaks (in a PA distribution observed at a given pulse longitude).

In the course of pulsar rotation, $\psi_{\text {in }}$ is changing with the pulse longitude. When $\vec{E}$ coincides with the dashed intermode separatrix (i.e. $\psi_{\text {in }}=45^{\circ}$ ), the orthogonal mode jump at maximum $V$ occurs. The maximum degree of circular polarisation that is observed at the PA jump, depends on the peak position and width of $N_{\Delta \phi}$. The step-wise PA curve of B1913+16 (Weisberg \& Taylor 2002), with the OPM transitions at peaks of $V$, can be interpreted in this way (cf. figs 1 and 7 in D17). The application of the model to the loop-shaped core PA distortions (Mitra et al. 2016), and to the $45^{\circ}$ PA jump, is also described in D17.

The research was funded by the National Science Center grant DEC-2011/02/A/ST9/ 00256. Participation in the conference was covered by NCAC.

\section{References}

Dyks, J. 2017, MNRAS, in press (D17)

Mitra, D., Rankin, J., \& Arjunwadkar, M. 2016, MNRAS, 460, 3036

Weisberg, J. M., Taylor J. H. 2002, ApJ, 576, 942 